\title{
The nucleoprotein of severe fever with thrombocytopenia syndrome virus processes a stable hexameric ring to facilitate RNA encapsidation
}

\author{
Honggang Zhou ${ }^{1,2^{*}}$, Yuna Sun4 ${ }^{*}$, Ying Wang ${ }^{5}$, Min Liu ${ }^{1,5}$, Chao Liu ${ }^{1,5}$, Wenming Wang ${ }^{1,5}$, Xiang Liu ${ }^{5}$, \\ Le $\mathrm{Li}^{1,5}$, Fei Deng ${ }^{3}$, Hualin Wang ${ }^{3 凶}$, Yu Guo ${ }^{1 \bowtie}$, Zhiyong Lou ${ }^{2 \bowtie}$ \\ ${ }^{1}$ College of Pharmacy and State Key Laboratory of Medicinal Chemical Biology, Nankai University, Tianjin 300071, China \\ ${ }^{2}$ Laboratory of Structural Biology and MOE Laboratory of Protein Science, School of Medicine and Life Sciences, Tsinghua \\ University, Beijing 100084, China \\ ${ }^{3}$ State Key Laboratory of Virology, Wuhan Institute of Virology, Chinese Academy of Sciences, Wuhan 430071, China \\ ${ }^{4}$ National Laboratory of Macromolecules, Institute of Biophysics, Chinese Academy of Science, Beijing 100101, China \\ ${ }^{5}$ High-throughput Molecular Drug Discovery Center, Tianjin Joint Academy of Biotechnology and Medicine, Tianjin 300457, China \\ $\bowtie$ Correspondence: louzy@xtal.tsinghua.edu.cn (Z. Lou), guoyu@nankai.edu.cn (Y. Guo), h.wang@wh.iov.cn (H. Wang) \\ Received April 8, 2013 Accepted April 16, 2013
}

\section{ABSTRACT}

Severe fever with thrombocytopenia syndrome virus (SFTSV), a member of the Phlebovirus genus from the Bunyaviridae family endemic to China, is the causative agent of life-threatening severe fever with thrombocytopenia syndrome (SFTS), which features high fever and hemorrhage. Similar to other negative-sense RNA viruses, SFTSV encodes a nucleocapsid protein (NP) that is essential for viral replication. NP facilitates viral RNA encapsidation and is responsible for the formation of ribonucleoprotein complex. However, recent studies have indicated that NP from Phlebovirus members behaves in inhomogeneous oligomerization states. In the present study, we report the crystal structure of SFTSV NP at $2.8 \AA$ resolution and demonstrate the mechanism by which it processes a ringshaped hexameric form to accomplish RNA encapsidation. Key residues essential for oligomerization are identified through mutational analysis and identified to have a significant impact on RNA binding, which suggests that correct formation of highly ordered oligomers is a critical step in RNA encapsidation. The findings of this work provide new insights into the discovery of new antiviral reagents for Phlebovirus infection.

KEYWORDS SFTSV, nucleoprotein, oligomer, RNP assembly, crystal structure

\section{INTRODUCTION}

Severe fever with thrombocytopenia syndrome (SFTS), a lifethreatening illness associated with high fever and hemorrhage, is endemic in eastern and central China (Stone, 2010; Yu et al., 2011). Severe fever with thrombocytopenia syndrome virus (SFTSV) is the causative agent of SFTS. SFTSV infection results in high fever, thrombocytopenia, leukocytopenia, gastrointestinal symptoms, neural symptoms, and bleeding with high mortality rates of up to $30 \%$ (Yu et al., 2011; Gai et al., 2012). The severity of SFTS results from an infection-induced cytokine storm with abnormally expressed cytokine profiles (Deng et al., 2012; Sun et al., 2012b). Up to 240 infections and 30 fatalities from SFTSV were reported in China in 2011 (Yu et al., 2011). However, to date, the molecular mechanism of SFTSV infection and replication, as well as its therapeutic management, has yet to be completely understood.

SFTSV is a member of the Phlebovirus genus of the Bunyaviridae family (Yu et al., 2011). Phylogenetic analysis shows that SFTSV is distant from the two other groups of Phlebovirus: the Sandfly fever group, which includes Rift Valley fever virus (RVFV), Punta Toro virus, Toscana virus, Massila virus, and Sandfly fever Sicilian virus, and the Uukuniemi group; this finding suggests that SFTSV is the prototype of a third group in Phlebovirus (Nichol et al., 2005; Cheng, 2011; Yu et al., 2011). Similar to all members of the Bunyaviridae family, the SFTSV genome contains tri-segmented negative-sense single-strand-

\footnotetext{
*These authors contributed equally to the work.
} 
ed RNAs (-ssRNAs), including large (L), medium (M), and small (S) segments (Yu et al., 2011). The $L$ segment encodes the RNA-dependent RNA polymerase (RdRp), the M segment encodes a precursor of glycoproteins, and the $S$ segment encodes both a nucleocapsid protein (NP) and a nonstructural protein using an ambisense coding strategy (Jiao et al., 2011).

Like other -ssRNA viruses, SFTSV contains ribonucleoprotein (RNP) complexes consisting of a genomic RNA wrapped by an NP (Jiao et al., 2011; Xuan et al., 2011). The genome length of the viral RNA (cRNA or viral genomic RNA) is only present in the form of an RNP throughout the entire virus replication cycle of a -ssRNA virus, either serving as a template for RNA synthesis or becoming packaged in the virion. Therefore, RNP assembly is a critical step in the replication of -ssRNA viruses (Ruigrok et al., 2011).

Negative-strand RNA virus NPs require two fundamental properties to function (Sun et al., 2012a): they must bind with the RNA virus and they must polymerize into large oligomers to protect the entire length of the viral genome RNA. However, the molecular architectures and biological functions of NPs encoded by Bunyaviridae members significantly differ from each other (Hastie et al., 2011; Qi et al., 2011; Guo et al., 2012). Two recent studies revealed that RVFV (Phlebovirus genus) NP oligomerizes with two distinct molecular architectures (Raymond et al., 2010; Ferron et al., 2011). The most significant difference between these two studies is the conformation of the $\mathrm{N}$ terminus of RVFV NP. In the dimerized RVFV NP of one study, the N-terminal arm (the first 30 amino acids) folds backward toward the body of the molecule, forming a compact architecture. This same $\mathrm{N}$-terminal arm extends to the adjacent molecule and forms a ring-shaped architecture in another study. The structure of RVFV NP bound to RNA has previously been published, and crystal structures and EM visualization of both the viral and reconstituted RNPs provide a better understanding of the process by which phleboviruses encapsidate their RNA genomes (Raymond et al., 2012). Thus, to gain further information on the NP encoded by members of the Bunyaviridae family, especially members of different groups within Phlebovirus, we determined the crystal structure of the SFTSV NP in this work. Our findings provide novel insights into NP oligomerization to the regulation of RNP assembly in the Bunuyaviridae family.

\section{RESULTS}

The recombinant SFTSV NP oligomerizes and binds cellular RNA

Binding with RNA and polymerization to protect the bound RNA against external nuclease are the key features of virally encoded NPs. The full-length SFTSV NP (residues M1-L245) was expressed in $E$. coli to investigate the structural details of the NP. The NP was purified under non-denaturing conditions to verify its natural features. The retention volume of the major peak ranged from $8 \mathrm{~mL}$ to $14 \mathrm{~mL}$ (Fig. 1), while that of the small peak was about $22 \mathrm{~mL}$, which corresponds to a molecu-

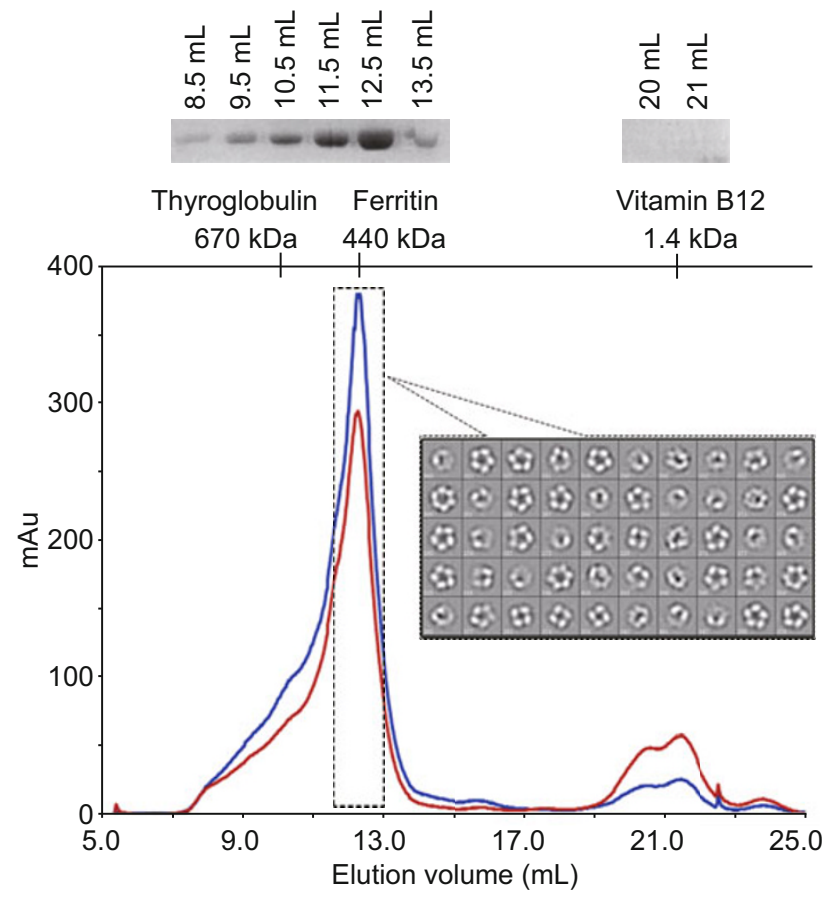

Figure 1. Size exclusion chromatograph of SFTSV NP without nuclease treatment. The E. coli expressing SFTSV NP $(1 \mathrm{mg})$ without nuclease pre-treatment was loaded onto a Superdex-200 HR 10/30 column. The retention volume of the highest peak is $12 \mathrm{~mL}$. Retention volumes for molecular weight standards are shown above. SDS-PAGE analysis of SEC elution fractions corresponding to the peaks is shown in the upper panel. Elution volumes of the various fractions are indicated above. The panel inside the dotted line shows representative class averages of SFTSV NP oligomers purified in solution. The side length of each square is $16 \mathrm{~nm}$, and the numbers of each representative class in all 16,492 particles are label in the left footnote.

lar weight of about $1.4 \mathrm{kDa}$. SDS-PAGE analysis indicated that the major peaks contained the expected protein size of SFTSV NP $(26 \mathrm{kDa})$. The results suggest that no protein contamination occurred during purification and revealed that the recombinant SFTSV NP is an inhomogeneous high-order oligomer in solution. Since virally encoded NPs bind with nucleic acid, we measured the $A_{260} / A_{280}$ ratio of peaks determined during gel filtration. The $A_{260} / A_{280}$ ratio of the major peak is 1.23 , indicating binding of cellular nucleic acids with the recombinant SFTSV NP. An additional peak showed an $\mathrm{A}_{260} / \mathrm{A}_{280}$ ratio of 1.8. SDSPAGE and agarose gel results suggest that this additional peak represents a DNA contaminant (data not shown), which may be formed by a low-molecular weight nucleic acid host. No viral genome RNA was found in the expression host, and recombinant SFTSV NP could randomly bind cellular nucleic acids to form a highly ordered oligomeric state. Electron micrographs by negative stain were taken using the fraction of the first peak. This protein behaved as string-like oligomers, showing structures ranging from tetramers to hexamers. 
A

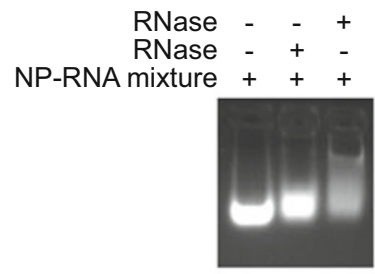

C

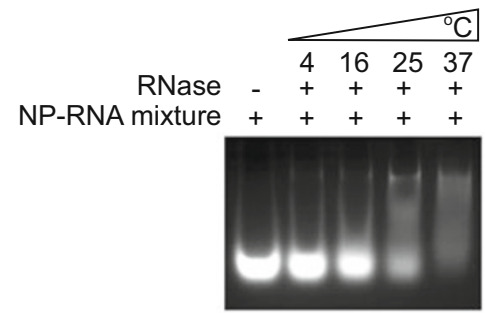

B

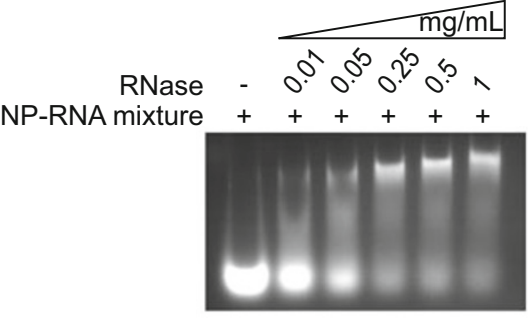

D

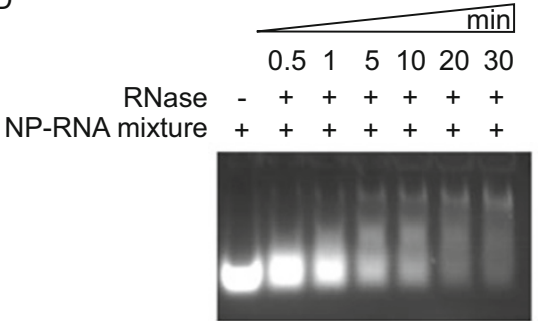

Figure 2. Nuclease digests of the SFTSV NP-RNA mixture. (A) A mixture of SFTSV was treated with DNase or RNase; (B) NP-RNA mixture treated with RNase at concentrations ranging from $0.01 \mathrm{mg} / \mathrm{mL}$ to $1 \mathrm{mg} / \mathrm{mL}$; (C) NP-RNA mixture treated with RNase at $4^{\circ} \mathrm{C}$, $16^{\circ} \mathrm{C}, 25^{\circ} \mathrm{C}$, or $37^{\circ} \mathrm{C}$; (D) NP-RNA mixture treated with RNase for $30 \mathrm{~s}$ to $30 \mathrm{~min}$.

\section{Removal of SFTSV NP-bound RNA by nuclease}

NPs encoded by non-segmented -ssRNA viruses tightly binded with RNA to form a continuous chain. While the NP-RNA complex is essentially resistant to any RNase digestion, NPs of the segmented -ssRNA viruses NP-RNA complex remain partially sensitive to such digestion (Ruigrok et al., 2011). Thus, we verified whether or not SFTSV NP-bound RNA is sensitive to exogenous nuclease.

Purified SFTSV NP-RNA was mixed with DNase or RNase at final concentrations ranging from $0.01 \mathrm{mg} / \mathrm{mL}$ to $1 \mathrm{mg} / \mathrm{mL}$ and then incubated for $30 \mathrm{~s}$ to $30 \mathrm{~min}$ at $4^{\circ} \mathrm{C}, 16^{\circ} \mathrm{C}, 25^{\circ} \mathrm{C}$, or $37^{\circ} \mathrm{C}$. The bound RNA was digested as shown in Fig. 2. The nucleic acid bound to SFTSV NP can be removed by RNase but not DNase, which indicates that the bound nucleic acid is mainly RNA, consistent with the biological function of a viral NP (Fig. 2A). The level of RNA degradation increased as the concentration of RNase increased from $0.01 \mathrm{mg} / \mathrm{mL}$ to $1 \mathrm{mg} / \mathrm{mL}$. Most of the bound cellular RNA was eliminated when the nuclease concentration reached $0.25 \mathrm{mg} / \mathrm{mL}$ (Fig. 2B). The bound RNA could be eliminated under $25^{\circ} \mathrm{C}$ and $37^{\circ} \mathrm{C}$ after incubation for $30 \mathrm{~min}$; however, some of the RNA was retained in solution after digests at $4^{\circ} \mathrm{C}$ and $16^{\circ} \mathrm{C}$ (Fig. 2C). SFTSV NP was not stable under $37^{\circ} \mathrm{C}$, and clear precipitation was found during digestion. Since RNA digestion shows obvious time gradient effects, RNase digestion at $16^{\circ} \mathrm{C}$ with a nuclease concentration of $0.01 \mathrm{mg} / \mathrm{mL}$ for $10 \mathrm{~h}$ was performed to maintain the optimum features of the SFTSV NP solution (Fig. 2D).

As the viral NP is responsible for RNA encapsidation and protection against exogenous nuclease, our results are not surprising. The RNA in influenza virus RNP can be easily removed by external nucleases, which indicates that influenza virus NP cannot protect bound RNA (Ye et al., 2006). In contrast to the previous finding that VSV NP is resistant to nuclease digestion, RNA encapsidated by the VSV NP was recently demonstrated to be susceptible to degradation through treatment at higher concentrations $(1 \mathrm{mg} / \mathrm{mL})$ of RNase A and higher temperatures $\left(37^{\circ} \mathrm{C}\right)$ (Green et al., 2011). This result demonstrates that SFTSV NP is effective against external nucleases at low concentrations and that it undergoes a dynamic conformational change to release bound RNA. Since nuclease-treated SFTSV NP showed good solution properties, we used this protein for subsequent structural investigation.

\section{Overall structure of SFTSV NP}

The crystal structure of the full-length SFTSV NP was determined using the multi-wavelength anomalous dispersion (MAD) method and refined to a resolution of $2.8 \AA$ with a final $R_{\text {work }}$ value of $22.1 \%\left(R_{\text {free }}=27.7 \%\right.$ ) (Table S1). All of the residues fitted well with the electron densities obtained, indicating their high structural stability. The overall structure of SFTSV NP presents a ring-shaped hexameric architecture, with inner and outer radii of $25 \AA$ and $105 \AA$, respectively (Fig. 3A). Although two hexameric rings may be observed in the crystallographic asymmetric unit (Fig. S2), gel filtration results indicated that the hexameric structure, which is consistent with the homogeneous molecular weight, is the native form of NP in solution (Fig. S3).

Each protomer of SFTSV NP has a compact structure with an additional $\mathrm{N}$-terminal arm extension and features three key parts: an N-terminal arm (hereafter called $\mathrm{N}$-arm) (M1-D34), an N-lobe (P35-L111), and a C-lobe (P112-L245) (Fig. 3B). All of the monomer folding patterns are predominantly composed of $\alpha$-helices. The $\mathrm{N}$-arm presents a bent architecture with two a-helices, while the $\mathrm{N}$ - and $\mathrm{C}$-lobes consist of four and seven a-helices, respectively. Two hydrophobic cavities, cavityR and 
A

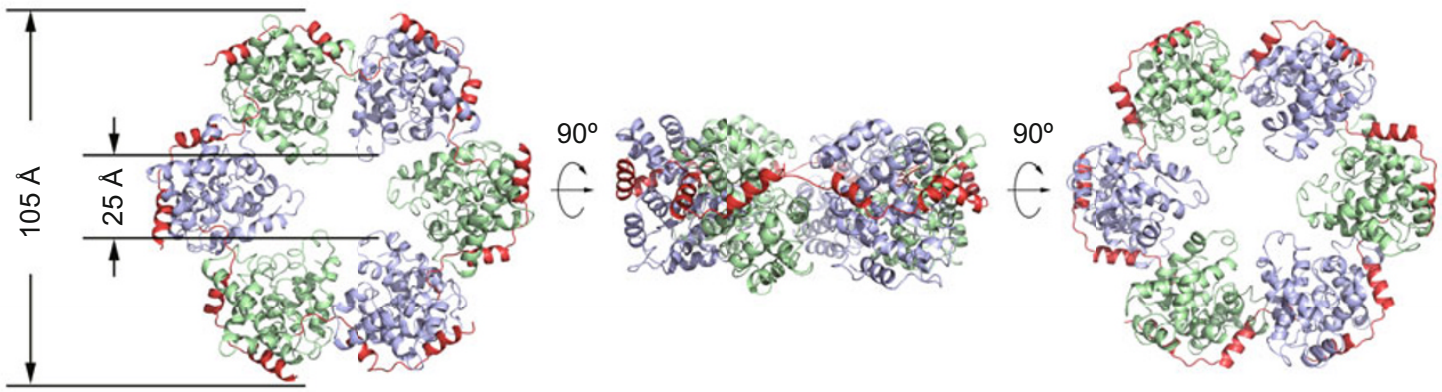

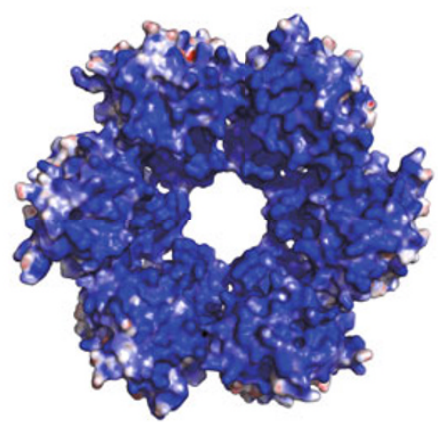

Top view
Side view
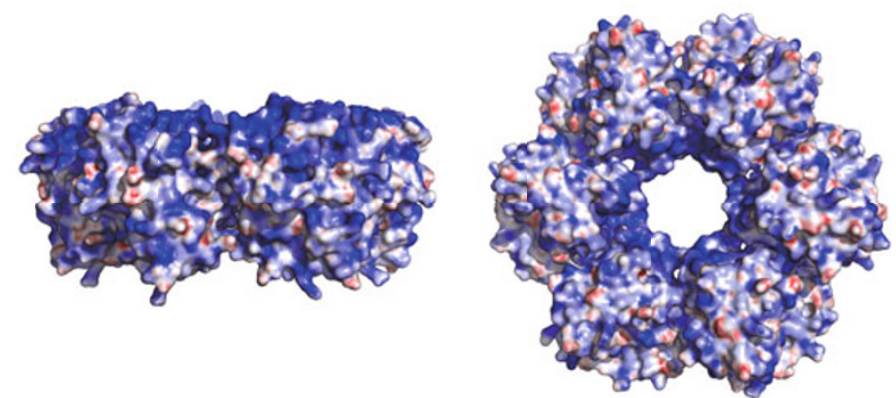

Bottom view

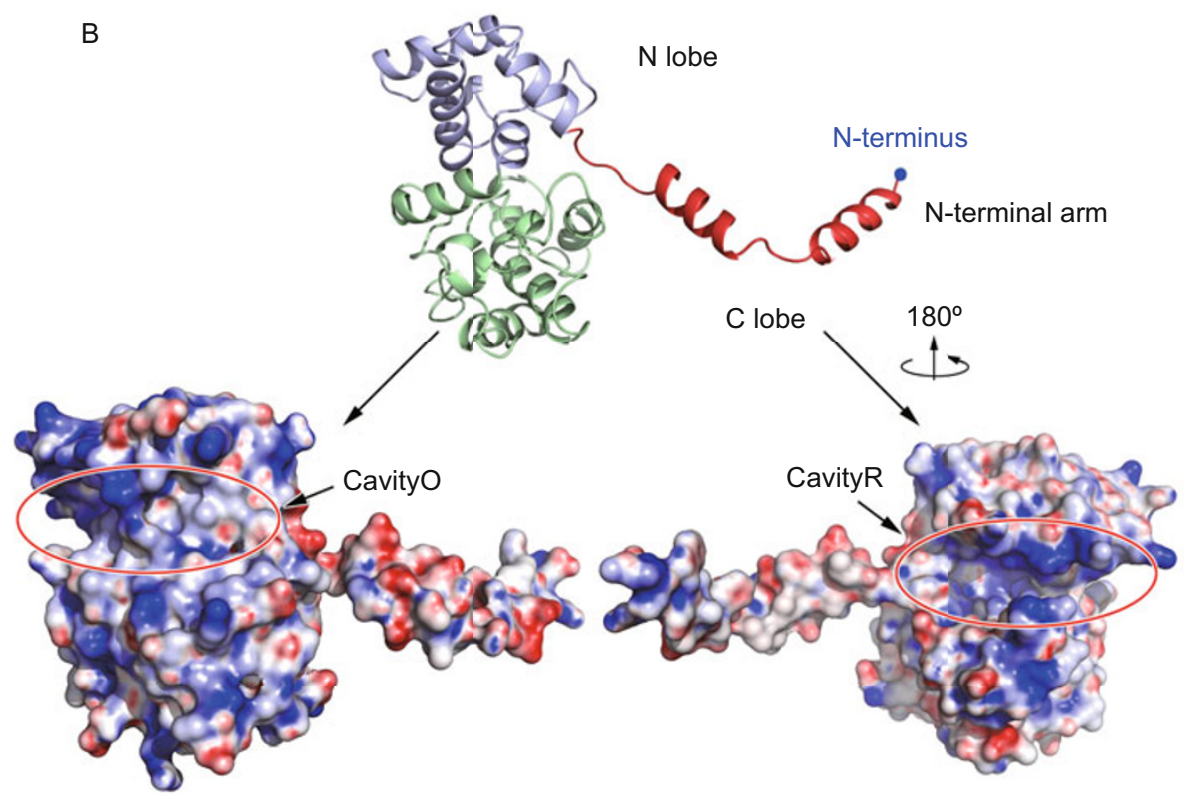

Figure 3. Structure of SFTSV NP. (A) The hexameric ring of SFTSV NP is shown as a ribbon and electron potential surface representation from the top, the side the bottom view. The molecules in the ribbon are presented as different colors in the upper panel. (B) Schematic diagram of the molecular architecture of the SFTSV NP monomer. The N-terminal arm, N lobe, and $\mathrm{C}$ lobe are indicated in red, blue, and green, respectively. The N-terminus is indicated by a small blue ball. The electron potential on the surface of the SFTSV NP monomer is shown with the same and the opposite orientation in the bottom panel. CavityO and cavityR are indicated by red circles.

cavityO, are observed. CavityR is a highly positively-charged putative RNA binding site located between the $\mathrm{N}$ - and $\mathrm{C}$-lobes, while cavity $\mathrm{O}$ is clamped by the $\mathrm{N}$ - and $\mathrm{C}$-terminal lobes at the inner and exterior sides of the hexameric ring, which accounts for the intermolecular interactions and oligomeric formation of NP. The N-arm rests in the hydrophobic cavityO of the adja- 
cent molecule and plays an essential role in oligomerization. The common feature of virally encoded NPs (Ye et al., 2006) is RNA binding, which is normally achieved through the interaction of positive-charged hydrophilic residues and the phosphate moiety of RNA. Residues such as R64, K67, K70, R95, R106, and K184 accomplish to electrostatic features of cavityR (Fig. S4).

\section{Intermolecular interactions}

The assembled RNP is composed of large amount of NP bound to the virus genomic RNA (Green et al., 2006). Each protomer of SFTSV NP interacts with adjacent molecules to maintain the hexameric architecture and protective stability of the NP oligomer (Fig. 4). The interactions between the adjacent molecules bury a total of $3132 \AA^{2}$ of the surface area as calculated by PISA (Krissinel and Henrick, 2007) according to the total $14,000 \AA^{2}$ surface of one molecule, which suggests strong interactions inside the hexameric ring. These interactions are primarily achieved by hydrophobic forces, van der Waals interactions, and hydrogen-bonding interactions (Table S2).

The interaction between the $\mathrm{N}$-terminal arm and a neighboring NP molecule (named Contacts I and II) is the most substantial interaction in the hexamer, and occupies nearly $50 \%$ of the total interaction surface $\left(1452 \AA^{2}\right)$. Contact I is formed by the first $\mathrm{N}$-terminal $\alpha$-helix of the $\mathrm{N}$-arm, from S5 to $\mathrm{L} 16$, and the first half of cavityO. A8, V9, I7, F11, and L16 face cavityO, providing the majority of the hydrophobic interaction. L21, F24 A25, L28, and A29 in the second helix of the N-arm hydrophobically interact with the other half of cavityO to contribute to oligomerization. Some side-to-side interactions (named Contacts III and IV) also assist in SFTSV NP oligomerization. The residues R99, R106, K164, and E192 come into contact with the residues $\mathrm{K} 74, \mathrm{~N} 77, \mathrm{~A} 131$, and $\mathrm{R} 186$ of a neighboring molecule through polar or electrostatic interactions.

Structure-based mutational analysis of the inter-monomer interface

Since the N-terminal arm and a subset of the residues in the body of the protein are associated with an adjacent molecule, we examined how substitutions on the interface affect the oligomerization state of SFTSV NP (Table 3). To exclude the impact of bound cellular RNA, all of the mutants were purified and subjected to nuclease treatment similar to that for the wildtype (WT) protein.

As the $\mathrm{N}$-arm contributes the majority of the intermolecular interaction, disruption of $\mathrm{N}$-arm-induced interactions is expected to destabilize SFTSV NP oligomerization. Therefore, a mutant lacking the first 34 residues of NP (hereafter named NP $\Delta 34$ ) was constructed. The recombinant NP $\Delta 34$ protein was overexpressed in $E$. coli, and gel filtration results indicated that the expected sizes of the proteins were obtained. Consistent with our hypothesis that the $\mathrm{N}$-arm is essential for oligomerization, SFTSV NP $\Delta 34$ eliminated all hexamers to monomers (Fig. 5A). Residues interacting with amino acids of electrostatic properties, such as alanine, aspartate, glutamate, and lysine, were substituted to elucidate their precise role in the N-arm. The mutants A8K, F11D, A25D, and L28K (Fig. 5B-E) could completely abolish the formation of ringshaped oligomers, whereas the mutants A8D, F11K, and L28E (Fig. $5 \mathrm{~F}-\mathrm{H}$ ) retained some of the hexamers. F24A and L28A (Fig. $5 \mathrm{I}$ and $5 \mathrm{~J}$ ) retained most of the hexamers and generated a very limited number of monomers. Other mutants in the $\mathrm{N}$-arm did not show obvious impacts on SFTSV NP oligomerization (Table S3).

Four side-to-side interactions were involved in the stabilization of SFTSV NP oligomers (Fig. 4). We replaced R99, R106, K164, R186, and E192 with alanine to investigate their impact on SFTSV NP oligomerization. Gel filtration results revealed that these mutants do not affect the oligomerization of SFTSV NP (Table S3). Our results indicate that hydrophobic interactions between the $\mathrm{N}$-arm and cavityO are mainly responsible for SFTSV NP oligomerization.

\section{Structure-based mutational analysis of the putative RNA} binding site

Although the structure of SFTSV NP with RNA has not been determined, the impacts of the residues on the putative RNAbinding site were analyzed. Considering that positively charged arginine/lysine residues interact with the negatively charged phosphate backbone of RNA molecules, we inferred that this positively charged region is likely responsible for the RNA binding of the SFTSV NP. We also constructed mutants on these sites to examine their RNA binding abilities compared with that of the SFTSV NP through the surface plasmon resonance (SPR) method using an 18-nt RNA probe. Single-site mutants included R64A, K67A, K70A, R95A, R106A, K184A, and R186A. SPR results showed that substitutions on single residues induce mild attenuations in binding affinity with the RNA probe compared with the WT protein. Since all of the single-site mutants showed similar impacts on RsNA binding, we used the R64A mutant to construct a representative curve (Fig. 6A). The triple-site mutant, R64A-K67A-K184A, abolished binding with the RNA probe. These results indicate that positively charged residues are involved in the RNA binding of SFTSV NP and that single substitution does not evidently affect this RNA binding activity.

\section{Disruption of SFTSV NP oligomerization impacts RNA binding}

While the positive charge inside cavityR is responsible for RNA binding, we also found that some oligomerization-defective mutants show limited RNA-binding during protein purification. We further analyzed the RNA-binding abilities of the mutants on the intermolecular interface to elucidate the impacts of SFTSV NP oligomerization on RNA binding. The Kd value calculated between the WT SFTSV NP and the18 nt RNA probe is $0.46 \mu \mathrm{mol} / \mathrm{L}$ (Fig. $6 \mathrm{~B}$ ) based on the SPR data.

The correct assembly of NP oligomers plays an essential 


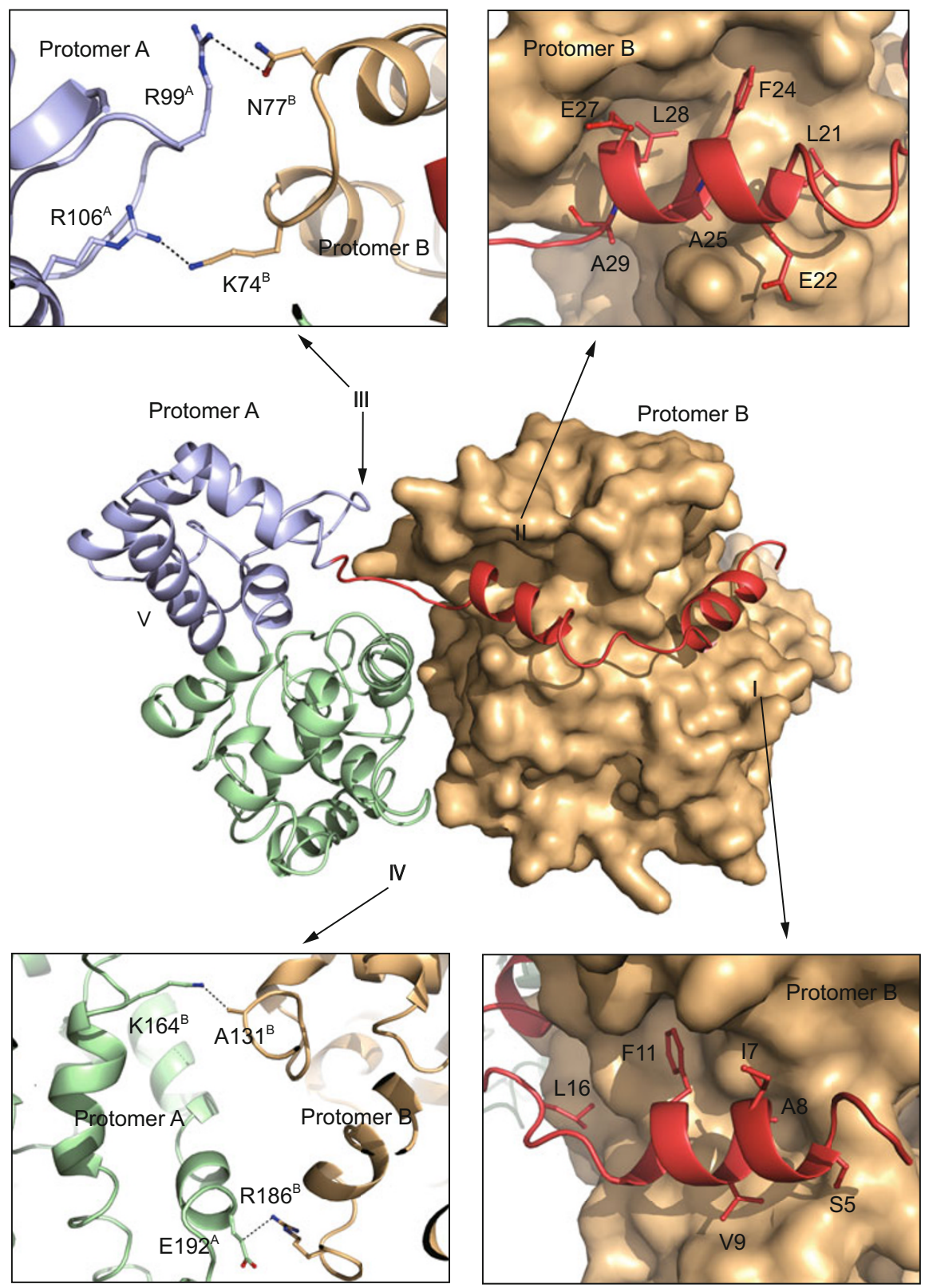

Figure 4. Inter-molecular interface of SFTSV NP. Two adjacent molecules were shown as ribbons (protomer A) and molecular surface (protomer B) diagram. Protomer A has the same color scheme shown in Fig. 4, and protomer B is covered as red surface. The Contacts I, II, III and IV were labeled and are detailed in the upper and bottom panels. Key residues, which account for inter-molecular interactions, are shown as colored sticks.

role in viral RNP formation and function. To test whether or not oligomerization of SFTSV NP is necessary for RNA packaging, we selected two mutants, L28E, which retains several oligomers, and F11D, which eliminates all oligomer. Consistent with our hypothesis, L28E showed a nearly 200 -fold decrease in RNA binding affinity compared with the WT protein (Fig. 6C). By contrast, F11D showed no detectable RNA-binding.

In the structural investigations of RVFV NP with different architectures, the RNA binding site is shadowed by the foldedback $\mathrm{N}$-arm and is unlikely to bind with RNA through this site
(Raymond et al., 2010). According to the primary sequence and structural similarities determined, the RNA-binding ability of SFTSV NP may not change significantly once cavityR is released from $\mathrm{N}$-arm. However, the mutant $\mathrm{NP} \triangle 34$, an oligomerization-defective mutant that features no $\mathrm{N}$-arm and a cavityR exposed to the solvent, showed no RNA-binding ability (Fig. 6A black line). This result suggests that correct oligomerization is crucial for RNA binding. All of our findings reveal that the formation of a correct oligomer is essential for SFTSV NP RNA encapsidation. 
A

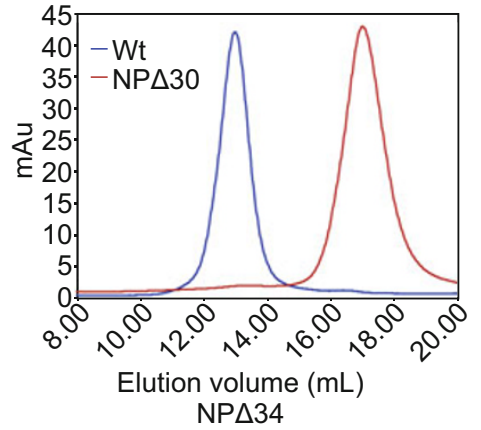

D

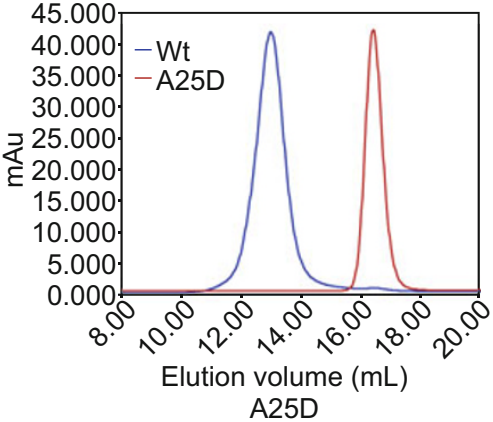

G

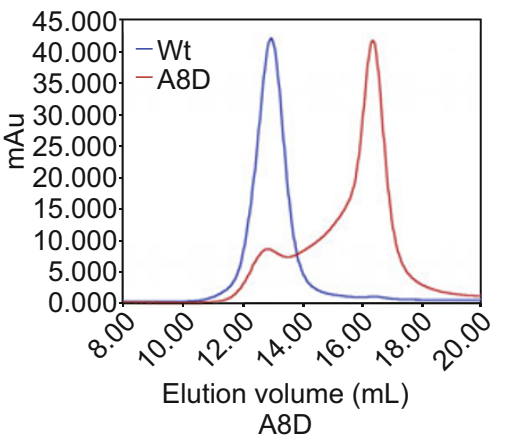

$\mathrm{J}$

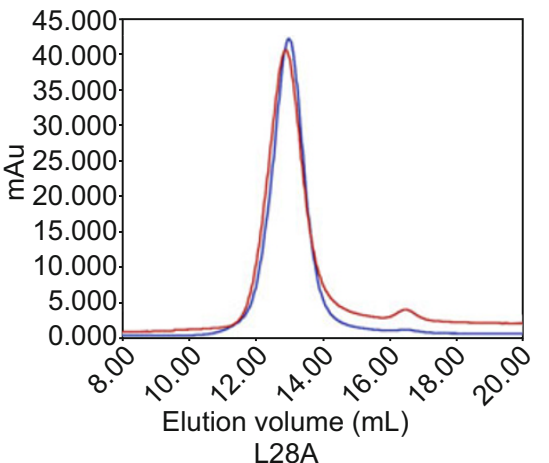

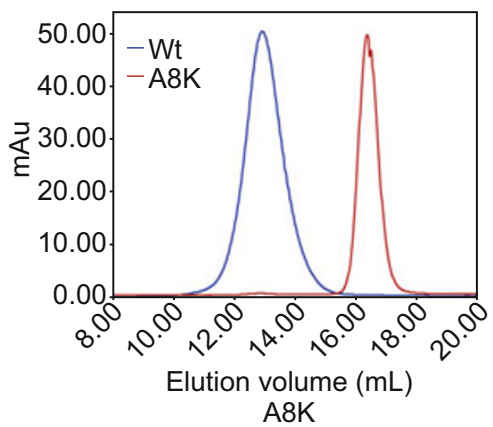

E

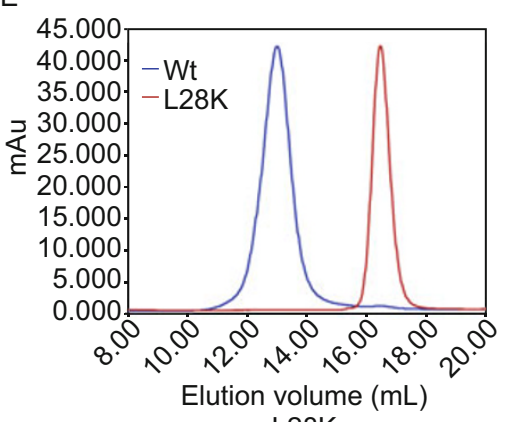

L28K

$\mathrm{H}$

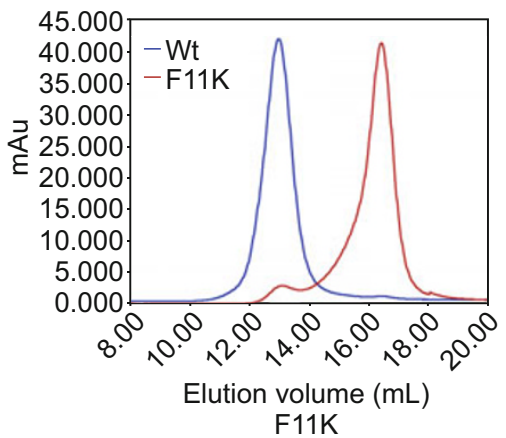

C

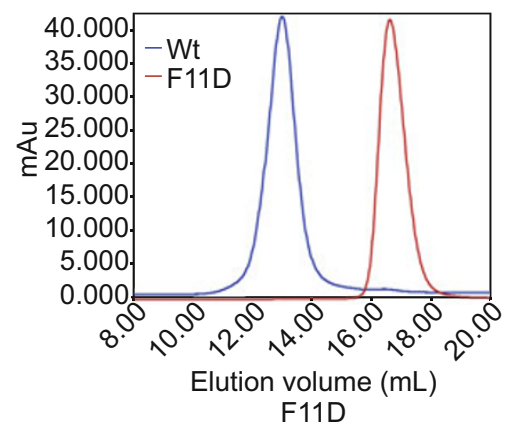

$\mathrm{F}$

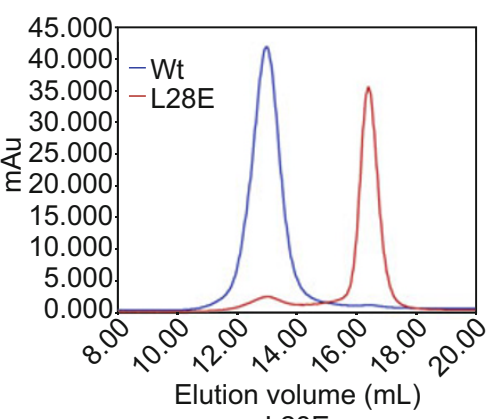

L28E

I

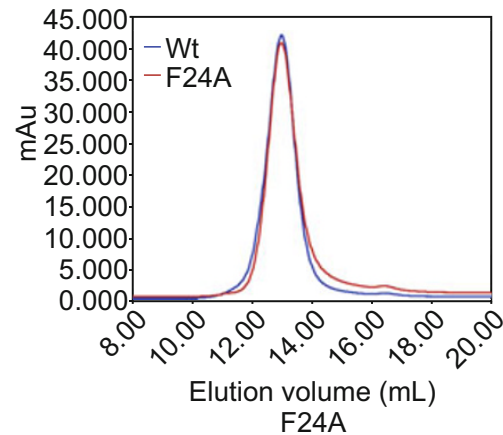

Figure 5. Mutational analysis of the inter-monomer interface. SEC of mutants that could eliminate or minimally abolish oligomerization of SFTSV NP. The WT NP and mutants on the inter-molecular interface ( $1 \mathrm{mg} / \mathrm{mL}$ each) were loaded onto a Superdex-200 HR $10 / 30$ column. Retention volumes of the hexamer and monomer are $13.3 \mathrm{~mL}$ and $16.4 \mathrm{~mL}$, respectively.

\section{DISCUSSION}

Phleboviruses facilitate a conserved dynamic mechanism of RNP formation

Although we do not get the similar monomeric or dimeric struc- ture of SFTSV NP under denature/renature condition like that of RVFV NP (Raymond et al., 2010), crystallographic analysis and primary sequence homology results between NPs of SFTSV and RVFV allow us to deduce that phleboviruses share a conserved dynamic mechanism of RNP formation. 
A

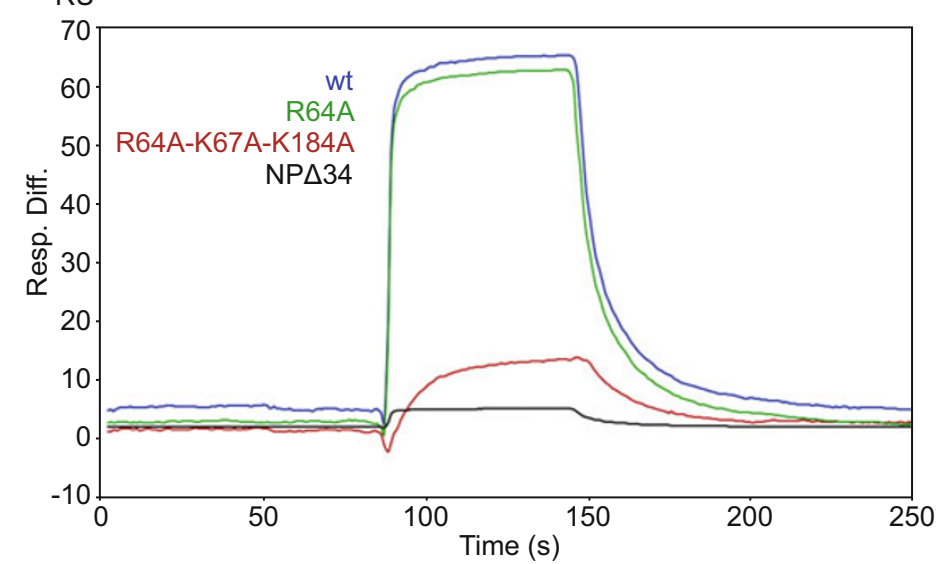

Figure 6. WT and mutational SFTSV NPs bind RNA with different affinities. Sensogram of the binding of RNA to immobilized WT SFTSV NP and mutants. (A) Comparison of the binding affinities of WT protein, the single-site mutant R64A, the triplesite mutant R64A-K67A-K184A, and the oligomerization defective mutant NP $\triangle 34$ to the RNA probe. The concentration of each protein is $8 \mu \mathrm{mol} / \mathrm{L}$. (B and C) Different concentrations of RNA were injected, and the signal $(R U)$ was plotted as a function of time. The colored curves represent the averages of three independent experiments, while the solid black lines represent the fitted curves. The binding affinities of the WT protein and L28E mutant were calculated in (B) and (C), respectively.
B

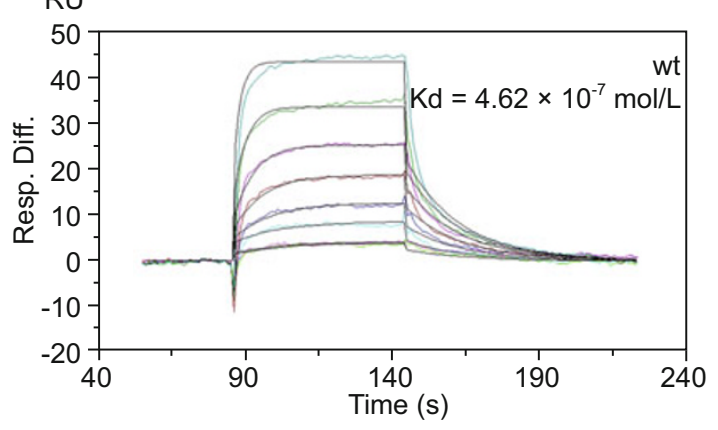

C

RU

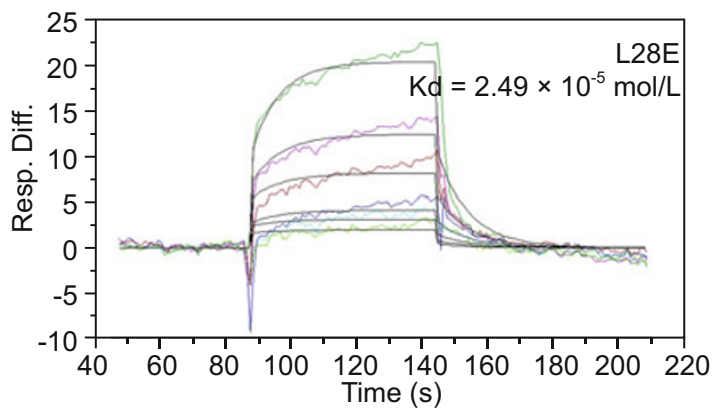

We determined four residues essential for the oligomerization of SFTSV NP, i.e., A8, F11, A25, and L28. Substitutions on these sites could significantly attenuate the oligomerization of the protein. Primary sequence alignment results show that the A8 residue is strictly conserved throughout three groups within phleboviruses (Fig. S1). Although the position on F11 residue in uukuniemi virus is an isoleucine residue, substitution of phenylalanine by isoleucine shows no impact on the oligomerization state. A similar observation is made on the position of the $\mathrm{L} 28$ residue. Although the mutants $\mathrm{L} 28 \mathrm{E}$ and $\mathrm{L} 28 \mathrm{~K}$ could demolish the oligomer formation of the SFTSV NP, the mutant L28F does not affect the formation of the ring-shaped complex. The position of A25 is not conserved in SFTSV and the viruses in two other groups. The alanine residue of SFTSV NP has no side chain, whereas the valine and isoleucine residues of RVFV and uukuniemi virus have hydrophobic side chains. However, substitutions of the A25 residue of the SFTSV NP by valine or isoleucine residues retain the oligomerization state of the protein, which suggests that mutants with a long hydrophobic side chain at this position are not critical for the formation of highly ordered oligomers. These results reveal that the ringshaped oligomerization of NP may be a conserved feature within phlebovirus members.

\section{Oligomerization plays a critical role in RNA encapsidation by SFTSV NP}

RNA encapsidation by NP is an essential step in -ssRNA repli- cation. The structures of several NPs of -ssRNA viruses, such as influenza virus (Ye et al., 2006; $\mathrm{Ng}$ et al., 2008), rabies virus (Albertini et al., 2006), VSV (Green et al., 2006), and BDV (Rudolph et al., 2003), have been reported. All of these NPs structurally have a deep positively-charged groove for RNA binding, although the direction of this groove is differs among nsNSV $\mathrm{NPs}$, resulting in distinct RNase sensitivity profiles ( $\mathrm{Ng}$ et al., 2008). These grooves are not obvious in the NPs of CCHFV and LAFV, indicating variations in RNA-binding within -ssRNA viruses (Guo et al., 2012).

While the SFTSV NP shows molecular folding patterns that differ from those of the NPs of VSV, rabies virus and influenza virus, a clear positively charged RNA-binding grove clamped by an $\mathrm{N}$-lobe and a $\mathrm{C}$-lobe indicates the similar functions of these NPs in RNA encapsidation. The cavityR of SFTSV NP comprises a set of positively charged residues (Fig. S4). The single-residue mutant cannot obviously attenuate the RNAbinding ability of SFTSV NP; by contrast, a multiple site mutant could abolish RNA-binding (Fig. 6A). This finding is also observed in the native form of structural homologues of RVFV NP. In addition, the RNA-binding groove in the monomeric structure of RVFV NP, which is obtained from the denaturation/renaturation method, is shadowed by the $\mathrm{N}$-arm and thus cannot bind with RNA (Ferron et al., 2011). We initially hypothesized that monomeric SFTSV NP mutants without the N-arm will expose the cavityR to the solvent and retain RNA-binding ability. Surprisingly, truncation of NP $\Delta 34$, which completely ex- 
poses the RNA binding site but abolishes the ring-shaped oligomerization of SFTSV NP, could not bind to the RNA in vitro (Fig. 6A). Oligomerization of SFTSV NP is essential for its RNA encapsidation since the cavityR of the NP $\Delta 34$ mutant stays in the released form. We further used L28E and F11D to test our speculation. L28E retained its RNA-binding ability, whereas F11D could not bind to RNA in the same conditions (Fig. 6C). All of these results demonstrate as well as the residues directly contribute to the RNA-binding, the residues responsible for oligomerization also plays essential role for RNA encapsidation.

In summary, we determined the hexameric ring-shaped crystal structure of SFTSV NP in native conditions. Besides significant functional variations in viral NPs among different -ssRNA virus families, the NP structures of viruses within the same viral family were found to be unidentical. For example, the structure and function of NPs from CCHFV and RVFV, both of which belong to the Bunyaviridae family, differ from each other significantly. Furthermore, the NP of RVFV displays notable structural shifts under different conditions. The structure of SFTSV NP reveals a conserved oligomerization state within Phlebovirus members. We also identified key residues, i.e., A8, $F 11, A 25$, and L28, which are crucial for the oligomerization of SFTSV NP. Mutational analysis of these residues revealed that they significantly attenuate the RNA-binding of SFTSV NP. A study on the truncated mutant NP $\Delta 34$, which demolishes oligomerization, also suggests that multimerization of SFTSV NP is essential for RNA binding. Furthermore, although cavityR in mutant NP $\triangle 34$ is sufficiently exposed to the solvent, its undetectable RNA binding indicates that the correct encapsidation of virus RNA requires not only a positively charged cavityR but also correct oligomerization brought about by the interaction between the $\mathrm{N}$-arm and cavity $\mathrm{O}$. Therefore, reagents, chemical compounds, or peptide mimics that could obstruct the interprotomer interactions of Phlebovirus NP may exert impacts on the correct process of RNA encapsidation and inhibit viral genome replication. This concept would be helpful in setting up a new strategy to discover antiviral reagents targeting Phlebovirus infections.

\section{MATERIALS AND METHODS}

\section{Protein production and crystallization}

The gene of the full-length SFTSV (strain HB29) NP (residues 1-245) was cloned into the pET-28a expression vector with a 6'His tag fused at the $\mathrm{N}$-terminus following a general protocol (Chen et al., 2011). The plasmid was transformed into E. coli strain BL21 (DE3), and transformed cells were cultured at $310 \mathrm{~K}$ in LB media containing $100 \mathrm{mg} / \mathrm{L}$ kanamycin. After the $\mathrm{OD}_{600}$ had reached 0.5 , the culture was cooled to $289 \mathrm{~K}$ and supplemented with $0.5 \mathrm{mmol} / \mathrm{L}$ isopropyl $\beta$-D-1thiogalactopyranoside. After overnight induction, the cells were harvested through centrifugation. The pellets were resuspended in lysis buffer containing $20 \mathrm{mmol} / \mathrm{L}$ HEPES (pH 6.8), $200 \mathrm{mmol} / \mathrm{L} \mathrm{NaCl}, 100$ $\mathrm{mmol} / \mathrm{L} \mathrm{MgCl} 2,100 \mathrm{mmol} / \mathrm{L} \mathrm{MnCl}_{2}, 50 \mu \mathrm{g} / \mathrm{mL}$ RNase, and $1 \mu \mathrm{g} / \mathrm{mL}$ DNase and homogenized in an ultra-high-pressure cell disrupter (JNBIO, Guangzhou, China) at $277 \mathrm{~K}$. The insoluble material was removed through centrifugation at $20,000 \mathrm{~g}$. The fusion protein was first purified by Ni-NTA affinity chromatography and elution with lysis buffer. The bound target protein was eluted with buffer containing $500 \mathrm{~mm}$ imidazole in $20 \mathrm{mmol} / \mathrm{L}$ HEPES (pH 6.8) and $200 \mathrm{mmol} / \mathrm{L} \mathrm{NaCl}$, further purified by a Hi-Trap heparin HP column (GE Healthcare) followed by a Superdex-200 gel filtration column (GE Healthcare), and dialyzed to buffer containing $20 \mathrm{mmol} / \mathrm{L}$ HEPES (pH 6.8) and $200 \mathrm{mmol} / \mathrm{L} \mathrm{NaCl}$. The SFTSV NP of over $95 \%$ purity was concentrated to $8 \mathrm{mg} / \mathrm{mL}$ and stored at $193 \mathrm{~K}$. SDS-PAGE analysis showed an $\mathrm{A}_{280} / \mathrm{A}_{260}$ ratio of 2.0.

Crystallization was performed at $291 \mathrm{~K}$ using the hanging-drop vapor-diffusion technique. Crystals were obtained by mixing $1 \mu \mathrm{L}$ of the protein solution (concentrated to $10 \mathrm{mg} / \mathrm{mL}$ ) with an equal volume of reservoir solution and then equilibrated against $300 \mu \mathrm{L}$ of the reservoir solution. Crystals of native SFTSV NP were obtained in a reservoir solution containing $100 \mathrm{mmol} / \mathrm{L}$ sodium malonate $(\mathrm{pH} 7.0)$ and $12 \%$ $(w / v)$ PEG 3350. Crystals with good diffraction quality grewto $100 \mu \mathrm{m} \times$ $100 \mu \mathrm{m} \times 200 \mu \mathrm{m}$ within $3 \mathrm{~d}$ in optimized well solution containing 100 $\mathrm{mmol} / \mathrm{L}$ sodium malonate $(\mathrm{pH} 7.0)$ and $10 \%(w / v)$ PEG 3350. Selenomethionine derivatives of SFTSV NP were purified following a general procedure (Ren et al., 2011) and crystallization with similar conditions as the native protein.

X-ray data collection, processing, and structure determination

All of the crystals were gradually transferred to a harvesting solution containing the precipitant solutions with added $15 \%(\mathrm{~V} / \mathrm{V})$ glycerol and then flash-frozen in liquid nitrogen for storage. Data were collected under cryogenic conditions at $100 \mathrm{~K}$.

Selenomethionine, a multi-wavelength anomalous dispersion (MAD) data set of SFTSV NP at the Se peak and inflection wavelengths, was collected at the Photon Factory (PF, Japan) beamline BL17A and processed using HKL2000 (Otwinowski and Minor, 1997). The crystals belonged to space group $P 2$ with cell parameters of $a=94.6 \AA, b=179.8 \AA, c=103.8 \AA, \alpha=\gamma=90^{\circ}$, and $\beta=96.6^{\circ}$. Six of the eight selenium atoms were located and refined, and MAD data phases were calculated and substantially improved by solvent flattening using the PHENIX program (Adams et al., 2002). A model was manually built into the modified experimental electron density using COOT (Emsley and Cowtan, 2004) and further refined in PHENIX.

Native data for SFTSV NP crystals were collected to $2.8 \AA$ at $100 \mathrm{~K}$ using an ADSC Q315 CCD detector on beamline BL17A in Photon Factory. The crystals belonged to the space group $P 2_{1}$ and showed slight differences in cell parameters with $a=102.2 \AA, b=104.4 \AA, c$ $=169.9 \AA, \alpha=\gamma=90^{\circ}$, and $\beta=100.3^{\circ}$. PHASER software (McCoy et al., 2007) was used to determine a correct solution via the molecular replacement method by using the model generated from MAD data as the initial search model. Several rounds of simulated annealing, restrained energy minimization, and individual B-factor refinement were conducted using PHENIX (Adams et al., 2002). Solvent molecules were located from stereochemically reasonable peaks in the $\sigma A-$ weighted $F_{O}-F_{C}$ difference electron density map. Final refinement statistics are summarized in Table S1. Model geometry was verified using the program PROCHECK (Laskowski et al., 1993). Structural figures were drawn using the program PyMOL (DeLano, 2002), and an electrostatic potential surface was generated by the Adaptive PoissonBoltzmann Solver (APBS) tool (Baker et al., 2001) incorporated into with PyMol. 


\section{SPR measurements}

The binding affinities of WT and mutant SFTSV NPs to an 18-nt RNA probe was studied using SPR on a Biacore 3000 instrument (GE Healthcare). The assays were carried out at $25^{\circ} \mathrm{C}$ in interaction buffer. To monitor protein-RNA interactions, the WT SFTSV NP and relevant mutants were immobilized to a CM5 sensor chip (GE Healthcare) by amine coupling until a surface density of 5000 response units (RU) had been achieved. The binding assays were performed in HBS buffer containing $20 \mathrm{mmol} / \mathrm{L} \mathrm{HEPES} \mathrm{(pH} \mathrm{6.8),} 200 \mathrm{mmol} / \mathrm{L} \mathrm{NaCl}$, and $0.005 \%$ $(v / v)$ Tween-20. The RNA probe [poly(rA), 18-nt, concentrations ranging from $0.25 \mu \mathrm{mol} / \mathrm{L}$ to $6.4 \mu \mathrm{mol} / \mathrm{L}$ ] was injected over the sensor chip in HBS buffer at a rate of $40 \mu \mathrm{L} / \mathrm{min}$. Data analysis and curves fitting were performed using BIA evaluation software (BIAcore)

\section{ACKNOWLEDGMENTS}

We thank the staff of Photon Factory for their generous help during diffraction data collection. This work was supported by the National Basic Research Program (973 Program) (No. 2013CB911103), the National Natural Science Foundation of China (Grant Nos. 31170678, 31170158, 31000332, and 81102374), and the Key Projects in the Tianjin Science and Technology Pillar Program (Grant Nos. 11ZCKFSY06900 and 11ZCKFSY06300).

\section{COMPLIANCE WITH ETHICS GUIDELINES}

Honggang Zhou, Yuna Sun, Ying Wang, Min Liu, Chao Liu, Wenming Wang, Xiang Liu, Le Li, Fei Deng, Hualin Wang, Yu Guo, and Zhiyong Lou declare that they have no conflict of interest.

This article does not contain any studies with human or animal subjects performed by the any of the authors.

\section{REFERENCES}

Adams, P.D., Grosse-Kunstleve, R.W., Hung, L.W., loerger, T.R., McCoy, A.J., Moriarty, N.W., Read, R.J., Sacchettini, J.C., Sauter, N.K., and Terwilliger, T.C. (2002). PHENIX: building new software for automated crystallographic structure determination. Acta Crystallogr D Biol Crystallogr 58, 1948-1954.

Albertini, A.A., Wernimont, A.K., Muziol, T., Ravelli, R.B., Clapier, C.R., Schoehn, G., Weissenhorn, W., and Ruigrok, R.W. (2006). Crystal structure of the rabies virus nucleoprotein-RNA complex. Science 313, 360-363.

Baker, N.A., Sept, D., Joseph, S., Holst, M.J., and McCammon, J.A. (2001). Electrostatics of nanosystems: application to microtubules and the ribosome. Proc Natl Acad Sci U S A 98, 10037-10041.

Chen, Q., Wang, Q., Xiong, L., and Lou, Z. (2011). A structural view of the conserved domain of rice stress-responsive NAC1. Protein Cell 2, 55-63.

Cheng, G. (2011). Innovator of in vitro virus culture-Dr. Chen-Hsiang Huang. Protein Cell 2, 782-783.

DeLano, W. (2002). The PyMOL molecular graphics system. DeLano Scientific, San Carlos, CA, USA.

Deng, B., Zhang, S., Geng, Y., Zhang, Y., Wang, Y., Yao, W., Wen, Y., Cui, W., Zhou, Y., Gu, Q., et al. (2012). Cytokine and chemokine levels in patients with severe Fever with thrombocytopenia syndrome virus. PLoS One 7, e41365.

Emsley, P., and Cowtan, K. (2004). Coot: model-building tools for molecular graphics. Acta Crystallogr D Biol Crystallogr 60, 2126-2132.
Ferron, F., Li, Z., Danek, E.I., Luo, D., Wong, Y., Coutard, B., Lantez, V., Charrel, R., Canard, B., Walz, T., et al. (2011). The hexamer structure of Rift Valley fever virus nucleoprotein suggests a mechanism for its assembly into ribonucleoprotein complexes. PLoS Pathog 7 , e1002030.

Gai, Z.T., Zhang, Y., Liang, M.F., Jin, C., Zhang, S., Zhu, C.B., Li, C., Li, X.Y., Zhang, Q.F., Bian, P.F., et al. (2012). Clinical Progress and Risk Factors for Death in Severe Fever with Thrombocytopenia Syndrome Patients. J Infect Dis 206, 1095-1102.

Green, T.J., Rowse, M., Tsao, J., Kang, J., Ge, P., Zhou, Z.H., and Luo, M. (2011). Access to RNA encapsidated in the nucleocapsid of vesicular stomatitis virus. J Virol 85, 2714-2722.

Green, T.J., Zhang, X., Wertz, G.W., and Luo, M. (2006). Structure of the vesicular stomatitis virus nucleoprotein-RNA complex. Science 313, 357-360.

Guo, Y., Wang, W., Ji, W., Deng, M., Sun, Y., Zhou, H., Yang, C., Deng, F., Wang, H., Hu, Z., et al. (2012). Crimean-Congo hemorrhagic fever virus nucleoprotein reveals endonuclease activity in bunyaviruses. Proc Natl Acad Sci U S A 109, 5046-5051.

Hastie, K.M., Liu, T., Li, S., King, L.B., Ngo, N., Zandonatti, M.A., Woods, V.L., Jr., de la Torre, J.C., and Saphire, E.O. (2011). Crystal structure of the Lassa virus nucleoprotein-RNA complex reveals a gating mechanism for RNA binding. Proc Natl Acad Sci U S A 108, 19365-19370.

Jiao, Y., Zeng, X., Guo, X., Qi, X., Zhang, X., Shi, Z., Zhou, M., Bao, C., Zhang, W., Xu, Y., et al. (2011). Preparation and evaluation of recombinant severe fever with thrombocytopenia syndrome virus nucleocapsid protein for detection of total antibodies in human and animal sera by double-antigen sandwich enzyme-linked immunosorbent assay. J Clin Microbiol 50, 372-377.

Krissinel, E., and Henrick, K. (2007). Inference of macromolecular assemblies from crystalline state. J Mol Biol 372, 774-797.

Laskowski, R., MacArthur, M., Moss, D., and Thornton, J. (1993). PROCHECK: a program to check the stereochemical quality of protein structures. J Appl Cryst 26, 283-291.

McCoy, A., Grosse-Kunstleve, R., Adams, P., Winn, M., Storoni, L., and Read, R. (2007). Phaser crystallographic software. J Appl Cryst 40, 658-674.

Ng, A.K., Zhang, H., Tan, K., Li, Z., Liu, J.H., Chan, P.K., Li, S.M., Chan, W.Y., Au, S.W., Joachimiak, A., et al. (2008). Structure of the influenza virus A H5N1 nucleoprotein: implications for RNA binding, oligomerization, and vaccine design. Faseb J 22, 3638-3647.

Nichol, S.T., Beaty, B.J., and Elliott, R.M. (2005). Family Bunyaviridae. In Virus taxonomy: classification and nomenclature of viruses, C.M. Fauquet, M.A. Mayo, J. Maniloff, U. Desselberger, and L.A. Ball, eds. (San Diego, CA, Elsevier Academic Press), pp. 695-716.

Otwinowski, Z., and Minor, W. (1997). Processing of X-ray diffraction data collected in oscillation mode. In Macromolecular Crystallography, part A, C.W. Carter Jr., and R.M. Sweet, eds. (Academic Press), pp. 307-326.

Qi, X., Lan, S., Wang, W., Schelde, L.M., Dong, H., Wallat, G.D., Ly, H., Liang, Y., and Dong, C. (2011). Cap binding and immune evasion revealed by Lassa nucleoprotein structure. Nature 468, 779-783.

Raymond, D.D., Piper, M.E., Gerrard, S.R., and Smith, J.L. (2010). Structure of the Rift Valley fever virus nucleocapsid protein reveals another architecture for RNA encapsidation. Proc Natl Acad Sci U S A 107, 11769-11774. 
Raymond, D.D., Piper, M.E., Gerrard, S.R., and Smith, J.L. (2012). Phleboviruses encapsidate their genomes by sequestering RNA bases. Proc Natl Acad Sci U S A 109, 19208-19213.

Ren, L., Qin, X., Cao, X., Wang, L., Bai, F., Bai, G., and Shen, Y. (2011). Structural insight into substrate specificity of human intestinal maltase-glucoamylase. Protein Cell 2, 827-836.

Rudolph, M.G., Kraus, I., Dickmanns, A., Eickmann, M., Garten, W., and Ficner, R. (2003). Crystal structure of the borna disease virus nucleoprotein. Structure 11, 1219-1226.

Ruigrok, R.W., Crepin, T., and Kolakofsky, D. (2011). Nucleoproteins and nucleocapsids of negative-strand RNA viruses. Curr Opin Microbiol 14, 504-510.

Stone, R. (2010). Infectious diseases. Rival teams identify a virus behind deaths in central China. Science 330, 20-21.

Sun, Y., Guo, Y., and Lou, Z. (2012a). A versatile building block: The structures and functions of negative-sense single-stranded RNA virus nucleocapsid proteins. Protein Cell 3, 893-902.

Sun, Y., Jin, C., Zhan, F., Wang, X., Liang, M., Zhang, Q., Ding, S., Guan, X., Huo, X., Li, C., et al. (2012b). Host cytokine storm is associated with disease severity of severe Fever with thrombocytopenia syndrome. J Infect Dis 206, 1085-1094.

Xuan, C., Shi, Y., Qi, J., Zhang, W., Xiao, H., and Gao, G.F. (2011). Structural vaccinology: structure-based design of influenza $A$ virus hemagglutinin subtype-specific subunit vaccines. Protein Cell 2, 997-1005

Ye, Q., Krug, R.M., and Tao, Y.J. (2006). The mechanism by which influenza A virus nucleoprotein forms oligomers and binds RNA. Nature 444, 1078-1082.

Yu, X.J., Liang, M.F., Zhang, S.Y., Liu, Y., Li, J.D., Sun, Y.L., Zhang, L., Zhang, Q.F., Popov, V.L., Li, C., et al. (2011). Fever with thrombocytopenia associated with a novel bunyavirus in China. N Engl J Med 364, 1523-1532. 